\title{
EFECTO INHIBITORIO DEL TRATAMIENTO MAGNÉETICO DE FRECUENGIA EXTREMADAMENTE BAJA SOBRE LA MICROBIÓTA TÍPICA DE MELAZA
}

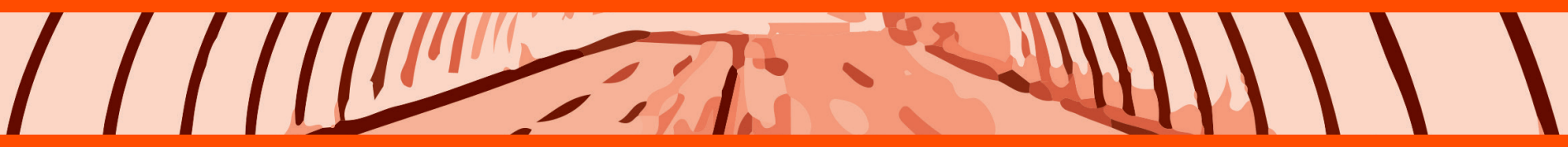

Autores:

\author{
Matilde Anaya Villalpanda ${ }^{1}$ \\ Tania Guzmán Armenteros ${ }^{2}$ \\ Alexander Vivar Pérez ${ }^{3}$ \\ Hilda Cobo Almaguer ${ }^{4}$ \\ Carlos Acea Fiallo 5
}

${ }^{1}$ Instituto de Investigaciones para la Industria Alimentaria (IIIA),
Carretera al Guatao, Lisa, La Habana, Cuba. (IIIA)
Email: matildea@quimica.cuaje.edu.cu / mavillal@iiia.edu.cu
Teléf.:(537) 202-0919, 202-0588, 202-0631, Fax: 537 24-6553

${ }^{2}$ Universidad Tecnológica Equinoccial, Km 4 1⁄2 vía Chone y Ave. Italia,

Santo Domingo-Ecuador

Email: gatm7012935@ute.edu.ec / Teléf.: 0992219091

${ }^{3}$ Instituto de Investigaciones para la Industria Alimentaria (IIIA), km 3 1/2 Carretera al Guatao, Lisa, La Habana, Cuba

Email: alexvivar@iiia.edu.cu

Teléf.:(537) 202-0919, 202-0588, 202-0631, 202-0614, Fax: 537 24-6553

${ }^{4}$ Instituto de Investigaciones para la Industria Alimentaria (IIIA), km 3 11/2

Carretera al Guatao, Lisa, La Habana, Cuba

Email: hilda@iiia.edu.cu

Teléf.:(537) 202-0919, 202-0588, 202-0631, 202-0614, Fax: 537 24-6553

${ }^{5}$ Grupo Ecosol Hidráulico, Calle 3ra esq. 12 s/n, Miramar, Playa, Cuba

Email: acea@hidraulico.copextel.com.cu

Recepción/Received: 2013-08-22

Teléf.:(537)830-1454, Ext. 05, 07

Aceptación/Accepted: 2013-09-28

Publicado/Published: 2013-12-18

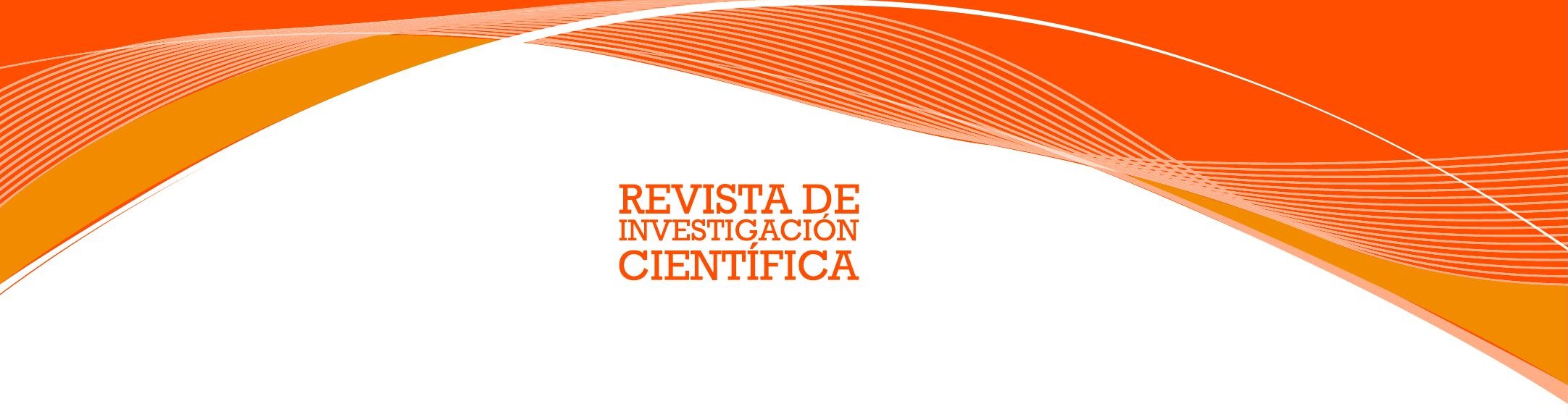




\section{Resumen}

La siguiente investigación tuvo por objetivo evaluar el efecto inhibitorio de un campo magnético oscilante (CMO)de baja frecuencia (6o Hz) y densidad $(6.5 \mathrm{mT})$ sobre la microbiota típica de melaza a diferentes concentraciones. Los factores experimentales fueron tiempo de tratamiento con CMO (0, 30 y $60 \mathrm{~min}$ ) y concentración de la melaza (12, 18 y $\left.24^{\circ} \mathrm{Bx}\right)$. La variable de respuesta a evaluar fue el recuento de mesófilos aerobios totales (conteo total) y de hongos y levaduras, por siembra en placas y dada en log ufc/mL. En general la disminución de los microorganismos no alcanzó la unidad logarítmica y se obtuvo una disminución de 0.5 \% respecto al control. Se observó la mayor incidencia sobre la microbiota en el orden: bacterias, levaduras y hongos filamentosos, siendo estos últimos los más resistentes al tratamiento magnético. El tiempo de tratamiento más efectivo fue 60 min en todos los grupos de microorganismos y fundamentalmente sobre la melaza a $12^{\circ} \mathrm{Bx}$. Se concluye que un valor bajo de densidad de CMO aplicado en idénticas condiciones a una muestra objeto de estudio puede tener efecto inhibitorio sobre los microorganismos indeseados que forman la microbióta típica de un determinado sustrato. Se demostró que no necesariamente existe una relación directa entre la densidad del campo magnético aplicado y la disminución del contenido microbiano.

Palabras clave: campo magnético oscilante, magnetorresistencia, tecnología emergente.

\section{Abstract}

The following research was aimed to evaluat of the inhibitory effect of an oscillating magnetic field (OMF) of low frequency (6o Hz) and density $(6.5 \mathrm{mT})$ on typical microbiota of molasses to different concentrations. The experimental factors were OMF treatment time ( 0 , 30 y 60 $\mathrm{min}$ ) and molasses concentration (12, 18 y $24^{\circ} \mathrm{Bx}$ ). Response variable to evaluate was the count of total aerobic mesophilic (total count) and of fungi and yeast, by culture in dishes and as in log cfu/mL. In general, microorganism decrease did not achieve the logarithmic unit and in a decrease of $0.5 \%$ compared to control was obtained. Higher incidence of microbiota in the order: bacteria, yeasts and filamentous fungi were observed, they were most resistance to magnetic treatment. The most effective treatment time in all groups of microorganisms was 60 min and fundamentally on dilution at $12^{\circ} \mathrm{Bx}$. Was concluded that an OMF low value density applied under identical conditions to a study sample may have inhibitory effect on undesirable microorganisms that form the typical microbiota of a given substrate. It was demonstrated that there is not necessarily a direct relationship between the applied magnetic field density and reduced microbial content.

Keywords: oscillating magnetic field, resistance of treatment magnetic, emergin techonology. 


\section{Introducción}

La melaza es un residuo de la industria azucarera y constituye un sustrato ideal para una gran variedad de microorganismos. Es una materia prima fundamental en la industria alcoholera y debe esterilizarse antes de inocular el fermento, y dicho proceso térmico tiene serias implicaciones tecnológicas, energéticas, económicas y medioambientales. Por tanto, cualquier estudio para hallar un método de inhibición, redundaría en una repercusión sobre dichas implicaciones. En tal sentido el tratamiento magnético (TM), clasificado como campo magnético estático (CME) u oscilante (CMO), surge como una tecnología emergente dado su poder inhibitorio (Barbosa, 2003).

Se conoce que entre la microbiota de las melazas se hallan bacterias como Bacillus subtilis, Bacillus cereus, Lactobacillus y Leuconostoc mesenteroides, el cual produce dextrana a partir de la sacarosa y provoca considerables pérdidas de ese azúcar (Rodríguez, 2005; Johany, Cuervo, Cárdenas, Durán, Mejía y Rodríguez, 2009). Los hongos y levaduras aparecen posteriormente puesto que no resisten los tratamientos térmicos del proceso de fabricación de azúcar (Rodríguez, 2005). En ese sentido, se informan resultados de estudios con TM sobre esos microorganismos.

Por ejemplo, al aplicar CME de $15 \mathrm{mT}$ (150 G) sobre Bacillus subtilis se observó un máximo de inhibición (Yoshimura, 1989) y cuando se empleó CMO de $50 \mathrm{~Hz}$ de $2 \mathrm{mT}$ (20 G) durante $10 \mathrm{~h}$ de exposición, hubo 16 \% de inhibición (Mehedintu y Berg, 1997). Por otra parte, algunos autores plantean que la acción repetida de la inducción magnética puede acelerar los procesos metabólicos en los hongos (González, 1999); sin embargo, se informó sobre la no significación de estas interacciones en levaduras (Chacón, Serguera y Rodríguez, 1999). Con respecto a la forma de aplicación de los TM, usualmente se aplican a las suspensiones microbianas en la fase inicial (Zapata, Hoyos y Moreno, 2005).

Por tanto, el siguiente estudio tuvo por objetivo evaluar el efecto inhibitorio del CMO de $60 \mathrm{~Hz}$ sobre la microbiota típica de melaza a diferentes concentraciones.

\section{Materiales y Métodos}

La composición de la melaza utilizada fue la siguiente:

\begin{tabular}{|l|l|l|l|}
\hline Azúcares totales & $53.03 \%$ & Reductores libres & $22.78 \%$ \\
\hline Azúcares fermentables & $46.53 \%$ & Cenizas & $9.30 \%$ \\
\hline Azúcares no fermentables & $6.48 \%$ & $\mathrm{pH}$ & 5.10 \\
\hline
\end{tabular}

Fuente: Manual de calidad del Banco de cepas del Instituto de Investigaciones para la Industria de los Alimentos

Se realizó un diseño experimental por el programa estadístico Design Expert ver. 6.0.1.0 (Stat- Ease, Minneapolis) con 12 corridas experimentales en un análisis de superficie respuesta D-óptimo de dos factores, los que se realizaron de forma aleatoria con CMO de 6.5 $\mathrm{mT}(65 \mathrm{G})$ y $60 \mathrm{~Hz}$ (Tabla I).

Tabla I. Niveles de los factores del diseño experimental.

\begin{tabular}{|c|cc|}
\hline \multirow{2}{*}{ Niveles } & \multicolumn{2}{c|}{ Factores } \\
\cline { 2 - 3 } & Tiempo (min) & Concentración (ox) \\
\hline 2 & 0 (nivel bajo) & 12 (nivel bajo) \\
& 60 (nivel alto) & 24 (nivel alto) \\
\hline
\end{tabular}

La variable de respuesta a evaluar fue el recuento de mesófilos aerobios totales (conteo total) y hongos y levaduras, dado en log ufc/mL. La determinación de la microbiota de la melaza se realizó empleando técnicas microbiológicas según las normas cubanas(ONNC, 2002; ONNC, 2005) mediante siembra en placas con agar para conteo en placas (mesófilos aerobios) y agar extracto de malta (hongos y levaduras). Las siembras en placas se realizaron por duplicado con previa dilución 10-6 con agua de peptona.

Los tratamientos de CMO se realizaron con un equipo experimental diseñado y confeccionado por Tecnologías Electrónicas 
(TECE) y caracterizado por Centro Nacional Electromagnetismo Aplicado (CNEA). El equipo consta de un generador de señal y una bobina con núcleo de aire.

Se realizaron dos corridas experimentales y los resultados se analizaron mediante ANOVA con el programa estadístico Statgraphics Centurion.

\section{Resultados y Discusión}

Se obtuvo un recuento inicial similar en todas las concentraciones para ambos grupos de microorganismos. Para el caso de hongos y levaduras, el mayor porcentaje fue para hongos filamentosos, con escasas levaduras. La disminución no alcanzó la unidad logarítmica, consecuente con lo informado que el CMO no elimina más que 3 ciclos log (Barbosa, 2003). Sin embargo, en todas las combinaciones se logró la disminución deseada, y en general, se obtuvo una inhibición de 0,3 a 0,5\% respeto al control.

A pesar de la similitud en el recuento inicial, la mayor incidencia fue sobre el grupo de hongos y levaduras (por la incidencia del TM sobre las levaduras). En el grupo de los mesófilos hubo un efecto notable en el crecimiento de las colonias de bacterias y levaduras, influyendo en su recuentro final.

Los factores tuvieron significación ( $\mathrm{p} \leq$ 0,05) destacándose que aumentó el efecto inhibitorio cuanto mayor fue el tiempo de exposición al CMO, en la medida que disminuyó la concentración de la melaza. Estos resultados concuerdan con lo observado al aplicar 100 $\mathrm{G}(10 \mathrm{mT})$ de $50 \mathrm{~Hz}$ por 24 min y los encontrados en trabajos similares al aplicar CMO de $60 \mathrm{~Hz}$ o menos (Yoshimura, 1989).

En las condiciones de estudio, 60 min fue más efectivo en los dos grupos de microorganismos y fundamentalmente sobre la dilución a $12{ }^{\circ} \mathrm{Bx}$. Los resultados obtenidos se muestran en la Tabla II.
Tabla II. Resultados obtenidos del recuento para cada grupo de microorganismo.

\begin{tabular}{|cccc|}
$\begin{array}{c}\text { Tiempo } \\
(\mathrm{min})\end{array}$ & $\begin{array}{c}\text { Concentración } \\
\left({ }^{\circ} \mathrm{Bx}\right)\end{array}$ & $\begin{array}{c}\text { Mesófilos aerobios } \\
(\log \mathrm{ufc} / \mathrm{mL})\end{array}$ & $\begin{array}{c}\text { Hongos y levaduras } \\
(\log \mathrm{ufc} / \mathrm{mL})\end{array}$ \\
\hline 30 & 12 & 6.16 & 6.37 \\
\hline 60 & 24 & 6.30 & 6.52 \\
\hline 60 & 18 & 6.25 & 6.46 \\
\hline 0 & 18 & 6.36 & 6.60 \\
\hline 60 & 24 & 6.34 & 6.58 \\
\hline 0 & 12 & 6.20 & 6.42 \\
\hline 0 & 12 & 6.23 & 6.48 \\
\hline 30 & 18 & 6.29 & 6.49 \\
\hline 60 & 12 & 6.09 & 6.31 \\
\hline 0 & 24 & 6.44 & 6.69 \\
\hline 60 & 12 & 609 & 6.62 \\
\hline 30 & 24 & 6.39 & \\
\hline
\end{tabular}

Nota: Las muestras controles son las que no recibieron tratamiento magnético (o min)

El procesamiento estadístico de las variables de respuesta en las variantes del diseño se ajustó a ecuaciones matemáticas. El análisis de varianza del modelo obtenido para el recuento demostró que el valor de $F$ (3117.31 y 656.67 para mesófilos y hongos, respectivamente) fue mayor que el de la tabla, por lo tanto se rechazó la hipótesis nula y se concluyó que el modelo fue significativo ( $\mathrm{p} \leq 0.05)$. La probabilidad de que los modelos cuadráticos $\left(R_{2}=0.9993\right.$ y 0.9967 para mesófilos y hongos, respectivamente) no expliquen satisfactoriamente el comportamiento de las variables fue muy baja, por lo cual se consideraron adecuados. En la Tabla III muestra los resultados del diseño y se aprecia que la variación del recuento de los hongos y mesófilos aerobios fue significativa $(p \leq 0.05)$ en relación con los factores tiempo de TM (A) y concentración de la melaza (B).

Tabla III. Resultados obtenidos del diseño de experimento.

\begin{tabular}{|ccccccc|}
\hline \multirow{2}{*}{ Factor } & \multicolumn{2}{c}{$\begin{array}{c}\text { Coeficientes } \\
\text { estimados }\end{array}$} & \multicolumn{2}{c}{$\begin{array}{c}\text { Desviación } \\
\text { estándar }\end{array}$} \\
\cline { 2 - 7 } & $\begin{array}{c}\text { Mesófilos } \\
\text { Aerobios }\end{array}$ & $\begin{array}{c}\text { Hongos y } \\
\text { Levaduras }\end{array}$ & $\begin{array}{c}\text { Mesófilos } \\
\text { Aerobios }\end{array}$ & $\begin{array}{c}\text { Hongos y } \\
\text { Levaduras }\end{array}$ & $\begin{array}{c}\text { Mesófilos } \\
\text { Aerobios }\end{array}$ & $\begin{array}{c}\text { Hongos y } \\
\text { Levaduras }\end{array}$ \\
\hline Intercepto & 6.30 & 6.50 & $2,19 \mathrm{E}+00$ & $5,18 \mathrm{E}+00$ & 0.0001 & 0.0001 \\
\hline $\mathrm{A}$ (Tiempo) & -0.060 & -0.071 & $1,03 \mathrm{E}+00$ & $2,43 \mathrm{E}+00$ & 0.0001 & 0.0001 \\
\hline $\mathrm{B}$ (Concentración) & 0.11 & 0.12 & $1,03 \mathrm{E}+00$ & $2,43 \mathrm{E}+00$ & 0.0001 & 0.0001 \\
\hline $\mathrm{A}^{2}$ & $-4,78 \mathrm{E}+00$ & -0.029 & $2,02 \mathrm{E}+00$ & $4,78 \mathrm{E}+00$ & 0.0554 & 0.0010 \\
\hline $\mathrm{B}^{2}$ & -0.028 & -0.010 & $2,02 \mathrm{E}+00$ & $4,78 \mathrm{E}+00$ & 0.0001 & 0.0721 \\
\hline $\mathrm{AB}$ & $9,53 \mathrm{E}+00$ & 0.016 & $1,17 \mathrm{E}+00$ & $2,77 \mathrm{E}+00$ & 0.0002 & 0.0013 \\
\hline
\end{tabular}


A partir de dichos resultados se obtuvo la siguiente ecuación:

$$
R M=X_{0}-X_{1} \cdot A+X_{2} \cdot B-X_{3} \cdot A^{2}-X_{4} \cdot B^{2}+X_{5} \cdot A B
$$

Donde RM: recuento de microorganismos; Xo: constante del modelo; $X_{1}, X_{2}, X_{3}, X_{4}$ y $X_{5}$ : coeficientes de regresión; A: tiempo (min) y B: concentración $\left({ }^{\circ} \mathrm{Bx}\right)$.

El signo negativo de $\mathrm{A}$ indica disminución del recuento a mayores valores de este factor. Teniendo en cuenta el mayor intercepto (6.50) y módulo del coeficiente (-0.071) de los hongos y levaduras, el efecto será mayor sobre éstos (específicamente sobre las levaduras, ya que los hongos filamentosos fueron más resistentes). Los signos positivos de los coeficientes de $B$ indican que se alcanzan mayores recuentos a niveles más altos de dicho factor, aunque muy similares entre ambos: 0.11 y 0.12 . Es decir, que a mayor concentración de la melaza el efecto inhibitorio del TM sobre los microorganismos es menor (Anaya, 2012) aunque es probable que los microorganismos queden atrapados entre los flóculos que forman las partículas coloidales debido a la alta ocurrencia de probabilidad de choques entre las mismas. En este caso una mayor concentración del medio se relaciona con mayor contaminación microbiológica inicial. Un comportamiento similar ocurre al analizar el efecto combinado de estos factores $(A B)$, sobre los hongos y levaduras.

No obstante, dichos factores aparecen en términos cuadráticos $\left(A^{2}\right.$ y $\left.B^{2}\right)$ con signo negativo, indicando que ambos provocan un efecto neto de disminución cuando aumentan sus niveles. En el caso de mesófilos aerobios A2 no fue significativo ( $p=0.0554$ ), lo cual indica una vez más que los mayores efectos del tiempo de TM son para el grupo de los hongos y levaduras. Sin embargo, con el término $B^{2}$ sucede lo contrario: no fue significativo para hongos y levaduras ( $p=0.0721$ ).

Por tanto, con estos resultados se demuestra que algunos géneros o especies de microorganismos también pudieran ser “magnetorresistentes". En este estudio la resistencia al TM aplicado se manifestó en el orden: bacterias, levaduras y hongos filamentosos.

\section{Conclusiones}

El crecimiento de la microbiota típica de la melaza se afectó al exponerla a CMO de baja frecuencia $(60 \mathrm{~Hz})$ y baja densidad $(6.5 \mathrm{mT})$. El efecto depende del tiempo de exposición (min) y la concentración de la melaza ( $\left.{ }^{\circ} \mathrm{Bx}\right)$. Se obtuvo $0.5 \%$ de inhibición respecto al control, observándose la mayor incidencia sobre la microbiota en el orden: bacterias, levaduras y hongos filamentosos.

En este estudio el tiempo de 60 min fue el más efectivo en todos los grupos de microorganismos, fundamentalmente sobre la dilución a $12{ }^{\circ} \mathrm{Bx}$. En futuras investigaciones se propone valorar otros tiempos, concentraciones y sustratos no incluidos en este estudio.

\section{Referencias}

Anaya, M. (2012). Tratamiento magnético como complemento tecnológico del proceso cervecero en la fábrica "Guido Pérez". Tesis presentada en opción al grado académico de Master en Ingeniería Alimentaria. Facultad de Química, ISPJAE. La Habana, Cuba.

Barbosa, GV (2003). Innovations in food processing. Technomic Publish Co. INC, Lancaster. Basel. Pennsylvania, USA. ISBN: $1-56676-782-2$.

Chacón D, Serguera M, Rodríguez Y (1999). Tratamiento magnético de un medio de cultivo utilizado en la propagación de la Candida utilis a nivel de zaranda. Tecnología Química 19 (3): 79-82.

González AR (1999). Estudio de la cinética de crecimiento en fase sumergida del biocontrolador Trichoderma viride bajo la influencia de campos electromagnéticos. Tecnología Química 19 (1): 67-71.

Johany Á, Cuervo R, Cárdenas H, Durán JA, Mejía LF, Rodríguez G (2009). Efecto de las concentraciones salinas en la inhibición de Leuconostoc mesenteroides en un ingenio azucarero del Valle del Cauca. Revista Científica Guillermo de Ockham, 7(1):13-18. 
Mehedintu M, Berg H (1997). Proliferation response of yeast Saccharomyces cerevisiae on electromagnetic field parameters. Bioelectroch. Bioenerg. 43, 67- 70.

Oficina Nacional de Normalización de Cuba. NC ISO 4833:2002. Microbiología de alimentos de consumo humano y animal. Guía general para la enumeración de microorganismos. Técnica de placa vertida a $30{ }^{\circ} \mathrm{C}$. Cuba.

Oficina Nacional de Normalización de Cuba. NC ISO 7954:2002. Microbiología de alimentos de consumo humano y animal. Guía general para la enumeración de levaduras y mohos. Técnica de placa vertida a $25^{\circ} \mathrm{C}$. Cuba.

Rodríguez E (2005). La dextranasa a lo largo de la industria azucarera. Biotecnología Aplicada; 22:11-19.

Yoshimura N (1989). Application of magnetic action for sterilization of food. Shokukin Kihatsu 24(3):46-48. Citado US FDA. Center for Food Safety and Applied Nutrition. June 2, 2000.

Zapata JE, Hoyos M, Moreno G (2005). Acción de un campo magnético sobre un cultivo aireado de Saccharomyces cerevisiae. Interciencia, 30 (7): 409-413. ISSN: 0378-1844. 